\section{Topical cyclosporine to control ocular surface disease in patients with chronic glaucoma after long-term usage of topical ocular hypotensive medications}

${ }^{1}$ Cornea \& Ocular Surface Services, Cataract \& Refractive Services, Dr R P Centre for Ophthalmic Sciences, All India Institute of Medical Sciences, New Delhi, India

${ }^{2}$ Glaucoma Services, Dr R P Centre for Ophthalmic Sciences, All India Institute of Medical Sciences, New Delhi, India

Correspondence: M Vanathi, Cornea \& Ocular Surface, Cataract \& Refractive Services, Dr R P Centre for Ophthalmic Sciences, All India Institute of Medical Sciences, New Delhi 110029, India Tel: +91 11 26593010; Fax: +911126588919. E-mail: vanathi_g@yahoo. com

Received: 3 June 2014 Accepted in revised form: 8 February 2015 Published online: 10 April 2015

Presented as a poster in the 4th Eucornea Congress, Amsterdam RAl, Netherlands, October 4-5, 2013.

\begin{abstract}
Purpose To evaluate changes in ocular surface and central corneal sub-basal nerve fiber layer (SBNFL) after topical cyclosporin therapy in chronic glaucoma patients on long-term topical antiglaucoma therapy. Methods A prospective comparative study of ocular surface evaluation of chronic glaucoma patients on long-term topical therapy treated concurrently with a topical cyclosporine $0.05 \%$ twice daily for 6 months and controls was done. The study parameters evaluated at recruitment and at the 6-month follow-up included details of topical antiglaucoma medications, visual acuity, intraocular pressure, ocular surface evaluation parameters (TBUT, Schirmers I, ocular surface staining scores and ocular surface disease (OSD) index score (OSDI)), central corneal sensation (Cochet Bonnett aesthesiometer), and central confocal microscopy to study the SBNFL density (SBNFLD).

Results Thirty-two eyes of 16 patients with chronic glaucoma and 30 eyes of 15 normal subjects as controls were studied. Mean TBUT, pre/post CsA treatment was $8.67 \pm 3.01 / 12.24 \pm 1.83 \mathrm{~s}(P=0.007)$. Mean conjunctival/corneal staining scores pre/post CsA treatment were $3.38 \pm 1.93 / 1.50 \pm 0.718$ $(P=0.00) / 5.19 \pm 1.82 / 1.81 \pm 0.78(P=0.098)$, respectively. Mean OSDI pre/post CsA treatment scores were $30.63 \pm 14.61 / 14.76 \pm 6.06$ $(P=0.007)$. Mean corneal sensations scores pre/post CsA treatment were $4.64 \pm 0.46 / 4.94 \pm 0.39(P=0.002)$. Central corneal SBNFLD pre and post CsA treatment was $8811.35 \pm 2985.29 / 10335.13 \pm 4092.064 \mu \mathrm{m} / \mathrm{mm}^{2}$ $(P=0.0001)$.
\end{abstract}

M Saini', R Dhiman'1 , T Dada², R Tandon ${ }^{1}$ and $M$ Vanathi ${ }^{1}$

Conclusions Schirmer's test, ocular surface staining scores, OSDI, corneal sensations, and corneal SBNFLD showed a statistically significant improvement following a 6-month concurrent topical CsA therapy.

Eye (2015) 29, 808-814; doi:10.1038/eye.2015.40; published online 10 April 2015

Introduction

Corneal innervation is vital for the maintainence of corneal epithelial integrity, proliferation function and in corneal wound healing after injury. ${ }^{1}$

The sub-basal nerve plexus along with stromal keratocytes secrete a number of neuropeptides, which facilitate cell mitogenesis and migration, DNA synthesis, neurite extension and survival, keratocyte proliferation, and the regulation of epithelial stem cells. ${ }^{2}$ These neuropeptides are diffusible factors that are believed to stimulate the epithelial growth, proliferation, differentiation, and the production of collagen type VII. 3,4

The epithelial cells, in reciprocation, produce the soluble factors neuronal growth factor and glial cell-derived neurotrophic factor with a neurotrophic effect. ${ }^{5}$ Chronic ocular surface inflammation sensitizes ocular surface nerves and can also disrupt corneal barrier function. Nerve degeneration that occurs in the scenario of chronic ocular surface inflammation, as in cases of dry eye, may lead to altered sub-basal nerve fiber morphology in eyes. ${ }^{6}$ Several adverse effects on ocular surface and corneal innervation has been reported in patients on long-term antiglaucoma medications. ${ }^{5}$ The role of in vivo confocal microscopy in ocular surface analysis of dry eye and glaucomatous patients has been 
elucidated..$^{5,7-14}$ Topical cyclosporine has been proven to have a beneficial effect in cases of dry eye disease (DED). ${ }^{15}$ In a recent study in rabbit eyes beneficial effects of topical cyclosporine has been demonstrated on adverse ocular surface changes produced by long-term antiglaucoma medications. ${ }^{16}$

The effect of topical cyclosporine therapy in chronic glaucoma patients on changes in the central corneal subbasal nerve fiber layer (SBNFL) density in human eyes has not been evaluated. The aim of this study was to evaluate the ocular surface changes and central corneal SBNFL changes in patients on long-term topical antiglaucoma medications induced by the concurrent use of topical cyclosporine therapy.

\section{Patients and methods}

This study was performed conforming to the Declaration of Helsinki. Informed consent was obtained from all the patients before enrollment in this prospective comparative, longitudinal open label study. The Institute Ethics Committee board approval was sought and obtained for the study. Patients on follow-up with the glaucoma clinic (during the period of January-December 2012), with chronic glaucoma on two or more topical antiglaucoma medications with preservatives for at least 6 months or more, and consenting to participate in the study were included in the study. Patients using combination therapy of topical antiglaucoma agents with BAK preservatives (timolol with brimonidine; ALPHAGAN ophthalmic solution $0.2 \%$, Allergan Inc., Irvine, CA, USA) one drop twice daily at 8 am and 8 pm daily with or without latanoprost (XALATAN latanoprost ophthalmic solution $0.005 \%$, Pfizer Inc., New York, NY, USA) one drop at bed time, were included in the study. Eyes with pre-existing ocular surface morbidities such as trachoma, cicatrizing conjunctivitis, history of intraocular surgery, laser treatment in recent 6 months, contact lens use, recent ocular inflammation/infection, autoimmune diseases, ocular surface diseases (OSDs) like dry eye due to other causes, previous, or current use of other ocular medications like artificial tear therapy were excluded from the study. Patients with previous history of using any other topical medications were excluded from the study. All patients recruited into the study were given topical CsA 0.05\% (Restasis, Allergan) one drop twice daily along with their current antiglaucoma treatment for a period of 6 months. Subjects with no ocular problems, who accompanied the glaucoma patients, were recruited as controls.

Demographic data including age, gender, and duration was obtained from all the patients. The baseline examination done at recruitment included visual acuity, intraocular pressure, ocular surface evaluation (tear break-up time (TBUT), Schirmer's I test, ocular surface staining score, OSD index (OSDI), central corneal sensation, and in vivo scanning slit confocal microscopy of the central cornea. Following the baseline evaluation, the patients were started on topical $0.05 \%$ cyclosporine therapy (Restasis, Allergan Inc) twice daily concurrent with the antiglaucoma medications. Six months following topical cyclosporine $0.05 \%$ therapy, the ocular surface evaluation (TBUT, Schirmer I test, ocular surface staining score, OSDI), central corneal sensation, and in vivo scanning slit confocal microscopy of the central cornea were repeated and the data were recorded on a predesigned proforma.

Clinical evaluation: comprehensive ocular examination was done for all the patients. Best corrected Snellen's visual acuity was recorded. Intraocular pressure was measured by Goldman applanation tonometry. Slit lamp biomicroscopic examination of anterior and posterior segment and ocular adnexa was done. OSDI ${ }^{17}$ score was calculated using a 12item OSDI questionnaire, Schirmer I test ${ }^{18}$ for tear production, fluorescein TBUT $^{18}$ for tear film stability, and the National Eye Institute ocular surface staining scores ${ }^{18,19}$ for ocular surface damage were evaluated.

Aesthesiometry: Central corneal sensation threshold was measured using Cochett Bonnet Aesthesiometer (CBA, Luneau, Paris, France) that stimulates the corneal nerves by direct contact. This was achieved by gently pressing the $0.12-\mathrm{mm}$ nylon thread against the anterior corneal surface. The longest thread $(6 \mathrm{~cm})$ was first used since it provides the lowest stimulus intensity. The examination was repeated by reducing the length in steps of $0.5 \mathrm{~mm}$ until the stimulus was felt. The criteria for the threshold were the filament length which gives a 50\% positive response from four stimuli presentations. Corneal sensation threshold was measured in length of nylon filament (in $\mathrm{cm}$ ). Manufacturer provided calibration table on conversion of nylon filament length to pressure exerted on eye was used to record the values.

Slit in vivo confocal microscopy of the cornea: scanning slit in vivo confocal microscopy (NIDEK technologies Srl, Italy Confoscan 4) of the central cornea to image the corneal SBNFL was performed in automatic gain mode using a standard setting of four passes with a scanning range of $200 \mu \mathrm{m}$ at $40 \mathrm{X}$ magnification to obtain central corneal imaging of the anterior cornea. The procedure was performed as follows. One drop of lubricating viscous gel was placed on the microscope objective piece and moved to a backward position. After topical anaesthesia and positioning of the patient's head on the adjustable headrest, the confocal microscope with the $40 \mathrm{X}$ objective was placed 1-1.5 $\mathrm{mm}$ above the apex of the corneal center. The patient was asked to look into the light, so that the optical center aligned with a lateral accuracy of probably $<1 \mathrm{~mm}$. Then the microscope was brought into optical contact with the cornea by manual 
advancement of the micrometer-controlled $\mathrm{Z}$ drive. From this point all further $x-y-z$ movements of the instrument were then controlled from the real time picture displayed on the monitor. Images were recorded and checked to ensure that the required images had been captured and were of sufficient quality for investigation. Each eye was scanned three times and the images were saved. At the time of analysis, two best images were selected for analysis, of which the best one containing maximum number of nerves imaged at the sub-basal plexus level was selected for analysis by a masked observer.

SBNFL were identified as unmyelinated fiber bundles consisting of straight and beaded fibers that course in the basal aspect of the basal epithelial cell layer. Confocal image analysis was performed by a masked observer unaware about demographic details of all recruited eyes. The nerve fibers were traced manually using Neuron J plugin of the available online public domain software Image J program (http:/ /www.imagescience.org/ meijering/software/neuronj), following which it automatically generates data regarding the total nerve number and total length/frame of sub-basal nerves. The SBNFL branches were counted as a separate nerve if the length of its visible portion was $<50 \mu \mathrm{m}$. The total number SBNFL was recorded as the mean number per scan for each cornea. The mean sub-basal nerve layer density was calculated as the total length of all the main nerves and their branches imaged divided by the area of the standard frame size $460 \mu \mathrm{m} \times 345 \mu \mathrm{m}$ containing images $\left(\right.$ area $\left.=0.16 \mathrm{~mm}^{2}\right)$.

Statistical analysis: clinical variables included for analysis were Schirmer I test, conjunctival staining score (NEI), corneal staining score (NEI), FTBUT, OSDI results, and corneal sensitivity. Two best imaged nerve fiber layer for each eye were considered for analyses of nerve fiber number, nerve length, and nerve density. Quantitative variables were expressed as mean and $\mathrm{SD}$, and the values obtained before and after topical cyclosporine $0.05 \%$ therapy were compared using two sample $t$-test. $P$-value $<0.05$ was considered statistically significant.

\section{Results}

A total of 32 eyes of 16 patients of mean age $47.24 \pm 16.21$ years (range: 22 to 75 years (10 male (mean age of $49.90 \pm 15$; range 23 to 75 years) and 6 female patients (mean age of $45 \pm 18$; range 30 to 55 years))) were eligible for topical cyclosporine therapy and further analysis. The control group comprised of 30 normal eyes of 15 subjects (age and sex matched) with normal eyes ((mean age $40.68 \pm 13.73$ years); males-15, mean age $41.43 \pm 10.80$ years; females-15, mean age $39.22+13.12$ years). The mean duration of topical hypotensives was $7.81 \pm 2.88$ years (range 0.6-12 years). Amongst the 16 patients, 11 had of bilateral primary open angle glaucoma, two had juvenile open angle glaucoma, and one of normal tension glaucoma, primary angle closure glaucoma and ocular hypertension (OHT) each. All recruited patients were using combination therapy of BAK preservativecontaining timolol $0.5 \%$ (one drop twice daily) and brimonidine $0.2 \%$ (one drop twice daily) along with or without prostoglandin analog-latanoprost $0.005 \%$ (one drop once a day). The number of glaucoma medications used was $2 \pm 0.5$. The mean IOP of all glaucoma patients was $16.74 \pm 5.47 \mathrm{~mm} \mathrm{Hg}$ and that of the controls was $13.52 \pm 1.78 \mathrm{~mm} \mathrm{Hg}$. All 16 patients had signs and symptoms of OSD with level 2-3 DED (DEWS classification ${ }^{20}$ ).

The pre CsA and post CsA treatment parameters are shown in Table 1. A statistically significant improvement was noted in the values of Schirmer I test, FTBUT, conjunctival staining scores, corneal staining scores OSDI, and corneal sensations before and post cyclosporine therapy.

The number of nerves and nerve length in study and control eyes on central corneal confocal microscopy of the SBNFL is given in Table 2. The mean SBNFL density of 30 eyes in the controls was $12273.15 \pm 3516.04 \mu \mathrm{m} / \mathrm{mm}^{2}$. The mean SBNFL density of 32 eyes before cyclosporine therapy was $8811.347 \pm 2985.285 \mu \mathrm{m} / \mathrm{mm}^{2}$. Following 6 months of topical cyclosporine $0.05 \%$ therapy there was a statistically significant improvement in the SBNFL

Table 1 Ocular surface evaluation parameters and corneal sensitivity pre/post cyclosporine therapy

\begin{tabular}{lcccr}
\hline & & Study group & \multicolumn{1}{c}{ Controls } \\
\cline { 2 - 4 } & Pre-CsA treatment & Post CsA treatment & P-value \\
\hline Schirmer's test (mm) & $7.28 \pm 3.48$ & $10.78 \pm 2.593$ & $(0.003)$ & $12.86 \pm 1.93$ \\
TBUT (s) & $8.67 \pm 3.01$ & $12.24 \pm 1.83$ & $(0.007$ & $11.80 \pm 1.88$ \\
Conjunctival staining scores & $3.38 \pm 1.93$ & $1.50 \pm 0.718$ & $(0.00)$ & $0.84 \pm 0.46$ \\
Corneal staining scores & $5.19 \pm 1.82$ & $1.81 \pm 0.78$ & $(0.098)$ & $1.10 \pm 0.58$ \\
OSDI & $30.63 \pm 14.61$ & $14.76 \pm 6.06$ & $(0.007)$ & $6.02 \pm 3.84$ \\
Corneal sensations (cm) & $4.64 \pm 0.46$ & $4.94 \pm 0.39$ & $(0.002)$ & $5.07 \pm 0.37$ \\
\hline
\end{tabular}

Pre-CsA, parameters before cyclosporine therapy.

Post CsA, parameters after cyclosporine therapy. 
Table 2 Central corneal SBNFL parameters in the study and the control group

\begin{tabular}{lccr}
\hline & Study group & Controls & P-value \\
\hline Nerve number & $3.58 \pm 0.99$ & $5.40 \pm 1.70$ & 0.001 \\
Nerve length & $1101.44 \pm 287.64$ & $1963.70 \pm 562.56$ & 0.001 \\
Nerve density & $8811.35+2985.29$ & $12273.15 \pm 3516.04$ & 0.001 \\
\hline
\end{tabular}

density (mean value of $10335.13 \pm 4092.064(P=0.0001)$ ) as shown in Figures 1a and b.

\section{Discussion}

OSD is a common comorbidity finding in glaucoma patients. Vision-related quality of life in patients on chronic long-term glaucoma therapy has been seen to be affected due to the co-existence of OSD and the chronic use of BAK-containing medications. ${ }^{17,21,22}$

Glaucoma patients show a prevalence of OSD in 59\% of those using more benzalkonium preservative-containing eye drops, ${ }^{21}$ and $47.6 \%$ of open angle glaucoma patients have been found to have OSD with a significant impact on visual function and quality of life. ${ }^{17}$ The prevalence of OSD in glaucoma patients increases with age $\mathrm{e}^{23}$ and can be compounded with the concurrent use of topical multidose hypotensive therapy containing preservatives. This toxicity has been associated with BAK, which is the most commonly used preservative and causes damage of the conjunctival and corneal epithelial cells and enhances the morbidity of the OSD. Long-term treatment with BAK-containing antiglaucoma medication, multidrop dosage, and beta blocker-containing formulations were found to be responsible for corneal toxicity in eyes on topical antiglaucoma treatment. ${ }^{24,25}$ The effects of glaucoma medications on corneal endothelium, keratocytes, and sub-basal nerves among participants in the OHT treatment study revealed that chronic topical hypotensive therapy resulted in a decrease in the number and density of corneal sub-basal nerve fiber bundles but did not affect the keratocyte density or corneal endothelial characteristics. ${ }^{14}$ Another retrospective study ${ }^{5}$ on the long-term effects of preservative-free and preservativecontaining antiglaucoma eye drops on the tear secretion and ocular surface had divided their study participants into five groups according to the type of topical hypotensive therapy and found that glaucomatous patients with chronic treatment show ocular surface alterations. The described confocal microscopy changes include significant decrease in density of superficial epithelial cells, higher density of basal epithelial cells, activated stromal keratocytes, higher beading of the nerves, lower number of sub-basal nerves and higher SBNFL tortuosity in those with ocular hypotensive medications with preservatives. ${ }^{14,26}$
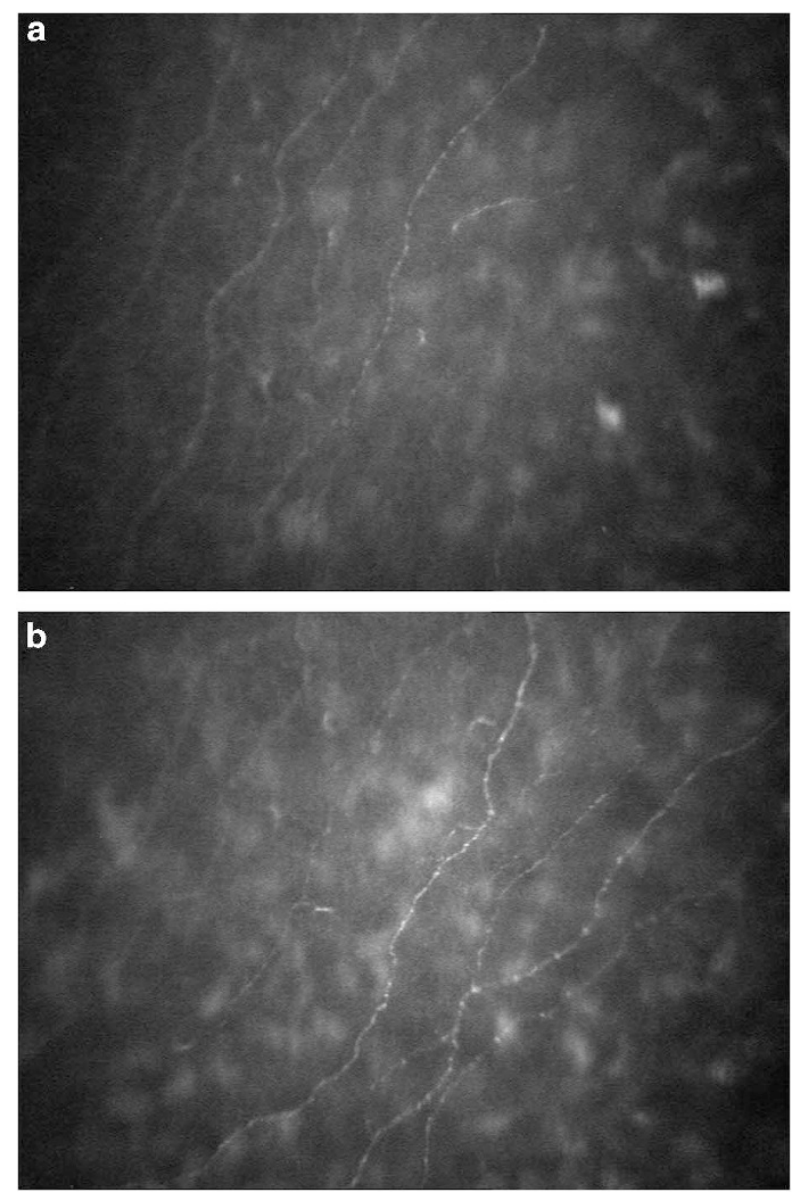

Figure 1 (a) Confocal microscopy imaging of the central cornea using NIDEK Confoscan 4 of the SBNFL showing decrease in the nerve number, branching and density of the SBNFL pretreatment with CsA. (b) Confocal microscopy imaging of the central cornea using NIDEK Confoscan 4 of the SBNFL showing increase in the nerve number, branching and density of the SBNFL post treatment with CsA.

Cyclosporine $0.05 \%$ is a topical immunomodulatory compound with anti-inflammatory properties that have been demonstrated to have a benefit in the treatment of dry eye. Topical hypotensive therapy with fixed combinations (prostaglandin analog and beta blocker eye drops) has been observed to increase the expression of the inflammatory markers HLA-DR and interleukin-6 with resultant deterioration in the ocular surface after three months of therapy. ${ }^{27,28}$ The anti-inflammatory effect of topical cyclosporine $0.05 \%$ administered twice daily has been proven to be beneficial to reduce the inflammatory effects of mild to moderate dry eye disease. ${ }^{15,20}$ Our study evaluated the concurrent effect of topical cyclosporine $0.05 \%$ therapy on the ocular surface in patients of long-term ocular hypotensives. Patients enrolled in our study were using combination therapy of topical timolol $0.5 \%$ and brimonidine $0.2 \%$ and prostaglandins analogs (with BAK preservatives). All recruited patients on long-term ocular 
hypotensive therapy had mild to moderate dry eye disease. Following 6 months of concurrent topical cyclosporine $0.05 \%$ therapy, there was a significant improvement noted in all ocular surface evaluation parameters (TBUT, Schirmer's test, conjunctival and corneal staining scores, and OSDI scores). This supports the beneficial effect of concurrent topical cyclosporine therapy in eyes on longterm ocular hypotensive medications.

The corneal nerve fibers are important for corneal trophism and for maintaining a healthy ocular surface. ${ }^{29}$ A lower nerve density may impair corneal sensitivity, ${ }^{6}$ as observed in glaucoma patients. A complex relationship seems to exist between the ocular surface changes, DED and decreased SBNFL in these eyes on chronic antiglaucoma medical treatment. ${ }^{5}$ Sensitization of ocular surface nerves and disruption of corneal barrier function occurs in chronic ocular surface inflammation due to DED leading to nerve degeneration and altered SBNFL characteristics. ${ }^{6}$ Studies show that topical administration of CsA $0.05 \%$ significantly increases tear production, decreases ocular surface damage and dry eye symptoms. ${ }^{30,31}$ Conjunctival epithelial biopsies from these patients show decreased molecular markers of immune activation and increased conjunctival goblet cell density. ${ }^{32,33}$ Topical cyclosporine therapy by its antiinflammatory effect results in a decrease in ocular surface inflammation and hence can have a beneficial effect on the corneal nerve regeneration. We therefore evaluated the change in nerve number, length and density of the central corneal SBNFL following 6 months of concurrent topical cyclosporine therapy in eyes on long-term ocular hypotensive therapy.

However, till date no study reported the effect of cyclosporine therapy on SBNFL density in chronic glaucoma patients. In our study we evaluated the changes in ocular surface evaluation parameters, corneal sensations, and SBNFL density following 6 months of cyclosporine therapy in chronic glaucoma patients on long-term topical hypotensive medications. A statistically significant improvement was noted in Schirmer test, TBUT, conjunctival and corneal staining scores, OSDI, and corneal sensations values upon 6 months of topical cyclosporine $0.05 \%$ therapy (Table 1 ).

In cases of glaucoma associated with significant OSD, although corneal staining severity was noted to be related to topical glaucoma therapy, OSDI was not seen as an ideal gauge of assessing OSD. ${ }^{34}$ However in our study, we found that a significant improvement in OSDI scores following 6 months of topical cyclosporine $0.05 \%$ twice daily therapy. This might be attributed to the fact that our study did not evaluate the effect of topical cyclosporine therapy on eyes with of glaucoma associated with significant OSD, but evaluated the effect of concurrent cyclosporine therapy in eyes with ocular hypotensive medications who had mild to moderate dry eye disease.

In normal corneas, the central corneal SBNFL density has been documented to be $10658 \pm 5581 \mu \mathrm{m} / \mathrm{mm}^{2}$ with NIDEK confoScan4 in vivo slit scanning confocal microscopy. ${ }^{35}$ In our control group of normal patients the mean central corneal SBNFL density was

$12273.15 \pm 3516.04$. In the study group, the SBNFL density of the central cornea of chronic glaucoma patients before cyclosporine therapy was $8811.35 \pm 2985.29 \mu \mathrm{m} / \mathrm{mm}^{2}$ that showed a significant improvement to $10335.13 \pm 4092.06 \mu \mathrm{m} / \mathrm{mm}^{2}$ after 6 months of concurrent topical cyclosporine $0.5 \%$ therapy.

Our study highlights a strong association of improvement of ocular surface evaluation parameters and SBNFL density with concurrent topical cyclosporine $0.05 \%$ therapy in eyes with chronic glaucoma on longterm topical hypotensive therapy, which show evidence of OSD due to drug molecule/BAK toxicity. The measurement of corneal sensation threshold with CochetBonnet aesthesiometer has certain drawbacks as stimulus reproducibility becomes difficult due to practical difficulties in alignment, placement, and replication of the force applied with the nylon filament.

In conclusion, it has been proven that patients with chronic glaucoma show significant ocular surface changes in affected eyes after prolonged usage of topical antiglaucoma medication, with altered morphology of sub-basal nerve fibers. Our study highlights that topical cyclosporine therapy seems to have beneficial effects in DED due to long-term topical hypotensive medications with improvement in ocular surface changes and central corneal SBNFL density. Concurrent topical cyclosporine $0.05 \%$ therapy may be considered in eyes with with DED due to long-term topical hypotensive therapy in chronic glaucoma patients as this has a beneficial effect on the ocular surface and corneal SBNFL.

\section{Summary}

\section{What was known before}

- Corneal innervation is vital for the maintenance of corneal epithelial integrity, proliferation, and in corneal wound healing. Ocular surface and Corneal SBNFL (reduced number and density) is adversely affected in glaucoma patients on long-term antiglaucoma medications (with preservatives). Beneficial role of cyclosporine has been proven in dry eye disease.

What this study adds

- Beneficial effects of cyclosporine therapy in patients on long-term antiglaucoma medications. Significant Improvement in ocular surface parameters. Significant increase in central corneal SBNFLD. First prospective comparative trial to study the effects of cyclosporine in glaucoma patients. 


\section{Conflict of interest}

The authors declare no conflict of interest.

\section{Acknowledgements}

This study was funded by the Institute Research Grant of All India Institute of Medical Sciences, New Delhi, India.

\section{References}

1 Herreras JM, Pastor JC, Calonge M, Asensio VM. Ocular surface alteration after long-term treatment with an antiglaucomatous drug. Ophthalmology 1992; 99(7): 1082-1088.

2 Beni'tez del Castillo JM, Wasfy MA, Fernandez C, Garcia-Sanchez J. An in vivo confocal masked study on corneal epithelium and subbasal nerves in patients with dry eye. Invest Ophthalmol Vis Sci 2004; 45(9): 3030-3035.

3 Garcia-Hirschfeld J, Lopez-Briones L, Belmonte C. Neurotrophic influences on corneal epithelial cells. Exp Eye Res 1994; 59(5): 597-605.

4 Baker KS, Anderson SC, Romanowski EG, Thoft RA, SundarRaj N. Trigeminal ganglion neurons affect corneal epithelial phenotype: influence on type VII collagen expression in vitro. Invest Ophthalmol Vis Sci 1993; 34(1): 137-144.

5 Martone G, Frezzotti P, Tosi GM, Traversi C, Mittica V, Malandrini A et al. An in vivo confocal microscopy analysis of effects of topical antiglaucoma therapy with preservative on corneal innervation and morphology. Am J Ophthalmol 2009; 147(4): 725-735.

6 Benítez-Del-Castillo JM, Acosta MC, Wassfi MA, Díaz-Valle D, Gegúndez JA, Fernandez C et al. Relation between corneal innervation with confocal microscopy and corneal sensitivity with noncontact esthesiometry in patients with dry eye. Invest Ophthalmol Vis Sci 2007; 48(1): 173-181.

7 Qazi Y, Aggarwal S, Hamrah P. Image-guided evaluation and monitoring of treatment response in patients with dry eye disease. Graefes Arch Clin Exp Ophthalmol 2014; 252(6): 857-872.

8 Villani E, Baudouin C, Efron M, Hamrah P, Kojima T, Patel SV et al. In vivo confocal microscopy of the ocular surface: from bench to bedside. Curr Eye Res 2014; 39(3): 213-231.

9 Villani E, Mantelli F, Nucci P. In-vivo confocal microscopy of the ocular surface: ocular allergy and dry eye. Curr Opin Allergy Clin Immunol 2013; 13(5): 569-576.

10 Villani E, Magnani F, Viola F, Santaniello A, Scorza R, Nucci P et al. In vivo confocal evaluation of the ocular surface morpho-functional unit in dry eye. Optom Vis Sci 2013; 90(6): 576-586.

11 Mastropasqua L, Agnifili L, Mastropasqua R, Fasanella V, Nubile $\mathrm{M}$, Toto L et al. In vivo laser scanning confocal microscopy of the ocular surface in glaucoma. Microsc Microanal 2014; 20(3): 879-894.

12 Mastropasqua L, Agnifili L, Fasanella V, Curcio C, Ciabattoni $\mathrm{C}$, Mastropasqua $\mathrm{R}$ et al. Conjunctival goblet cells density and preservative-free tafluprost therapy for glaucoma: an in vivo confocal microscopy and impression cytology study. Acta Ophthalmol 2013; 91(5): e397-e405.

13 Agnifili L, Fasanella V, Costagliola C, Ciabattoni C, Mastropasqua R, Frezzotti P et al. In vivo confocal microscopy of meibomian glands in glaucoma. Br J Ophthalmol 2013; 97(3): 343-349.

14 Baratz KH, Nau CB, Winter EJ, McLaren JW, Hodge DO, Herman DC et al. Effects of glaucoma medications on corneal endothelium, keratocytes, and subbasal nerves among participants in the ocular hypertension treatment study. Cornea 2006; 25(9): 1046-1052.

15 Zhou XQ, Wei RL. Topical cyclosporine A in the treatment of dry eye: a systematic review and meta-analysis. Cornea 2014; 33(7): 760-767.

16 Cho HK, Park MH, Moon JI. The effect of additional topical cyclosporin or vitamin A on the ocular surface during antiglaucoma medication administration. Ophthalmic Res 2012; 48: 139-145.

17 Skalicky SE, Goldberg I, McCluskey P. Ocular surface disease and quality of life in patients with glaucoma. Am J Ophthalmol 2012; 153: 1-9.

18 Nelson JD. In office diagnostic tests for dry eye disease. In: Asbell PA, Lemp MA (eds). Dry Eye Disease: The Clinician's Guide to Diagnosis and Treatment. Thieme Medical Publishers, Inc.: New York, NY, USA, 2006, pp 39-44.

19 Behrens A, Doyle JJ, Stern L, Chuck RS, McDonnell PJ, Azar DT et al. Dysfunctional tear syndrome study group. Dysfunctional tear syndrome: a Delphi approach to treatment recommendations. Cornea 2006; 25: 900-907.

20 The definition and classification of dry eye disease: Report of the Definition and Classification Subcommittee of the International Dry Eye Workshop (2007). Ocul Surf 2007; 5: 75-92.

21 Leung EW, Medeiros FA, Weinreb RN. Prevalence of ocular surface disease in glaucoma patients. J Glaucoma 2008; 17: 350-355.

22 Pisella PJ, Pouliquen P, Baudouin C. Prevalence of ocularsymptoms and signs with preserved and preservativefree glaucoma medication. Br J Ophthalmol 2002; 86(4): 418-423.

23 Kaštelan S, Tomić M, Metež Soldo K, Salopek-Rabatić J. How ocular surface disease impacts the glaucoma treatment outcome. Biomed Res Int 2013; 2013: 696328.

24 Lee S, Kim MK, Choi HJ, Wee WR, Kim DM. Comparative cross-sectional analysis of the effects of topical antiglaucoma drugs on the ocular surface. Adv Ther 2013; 30(4): 420-429.

25 Rosin LM, Bell NP. Preservative toxicity in glaucoma medication: clinical evaluation of benzalkonium chloridefree $0.5 \%$ timolol eye drops. Clin Ophthalmol 2013; 7: 2131-2135.

26 Iester M, Telani S, Frezzotti P, Motolese I, Figus M, Fogagnolo $\mathrm{P}$ et al. Ocular Surface changes in glaucomatous patients treated with and without preservatives beta-blockers. J Ocul Pharmacol Ther 2014; 30(6): 476-481.

27 Russ HH, Nogueira-Filho PA, Barros Jde N, de Faria NV, Montiani-Ferreira F, Gomes JÁ et al. Ocular surface evaluation in patients treated with a fixed combination of prostaglandin analogues with $0.5 \%$ timolol maleate topical monotherapy: a randomized clinical trial. Clinics (Sao Paulo) 2013; 68(10): 1318-1324.

28 Arici MK, Arici DS, Topalkara A, Guler C. Adverse effects of topical antiglaucoma drugs on the ocular surface. Clin Experiment Ophthalmol 2000; 28: 113-117.

29 Muller LJ, Marfurt CF, Kruse F, Tervo TM. Corneal nerves: structures, contents and function. Exp Eye Res 2003; 76: $521-542$. 
30 Sall K, Stevenson OD, Mundorf TK, Reis BL. Two multicenter,randomized studies of the efficacy and safety of cyclosporine ophthalmic emulsion in moderate to severe dry eye disease. CsA Phase 3 Study Group. Ophthalmology 2000; 107: 631-639.

31 Kymionis GD, Bouzoukis DI, Diakonis VF, Siganos C. Treatment of chronic dry eye: focus on cyclosporine. Clin Ophthalmol 2008; 2(4): 829-836.

32 Kunert KS, Tisdale AS, Gipson IK. Goblet cell numbers and epithelial proliferation in the conjunctiva of patients with dry eye syndrome treated with cyclosporine. Arch Ophthalmol 2002; 120(3): 330-337.
33 Pflugfelder SC, De Paiva CS, Villarreal AL, Stern ME. Effects of sequential artificial tear and cyclosporine emulsion therapy on conjunctival goblet cell density and transforming growth factor-beta2 production. Cornea 2008; 27(1): 64-69.

34 Mathews PM, Ramulu PY, Friedman DS, Utine CA, Akpek EK. Evaluation of ocular surface disease in patients with glaucoma. Ophthalmology 2013; 120(11): 2241-2248.

35 Patel DV, Tavakoli M, Craig JP, Efron N, McGhee CNJ. Corneal Sensitivity and Slit Scanning In Vivo Confocal Microscopy of the Subbasal Nerve Plexus of the Normal Central and Peripheral Human Cornea. Cornea 2009; 28: 735-740. 